\title{
Gravity Survey on the Mizuho Plateau, East Antarctica: SEAL Seismic Exploration in 2001-2002
}

\author{
Shigeru Toda ${ }^{{ }^{*}}$, Hiroki Miyamachi ${ }^{2}$, Masaki Kanao $^{3}$, Takeshi Matsushima ${ }^{4}$, \\ Masamitsu Takada ${ }^{5}$, Atsushi Watanabe ${ }^{6}$, Mikiya Yamashita ${ }^{7}$ \\ ${ }^{1}$ Department of Earth Sciences, Faculty of Education, Aichi University of Education, Kariya, Japan \\ ${ }^{2}$ Department of Earth and Environmental Sciences, Faculty of Science, Kagoshima University, Kagoshima, Japan \\ ${ }^{3}$ National Institute of Polar Research, Tokyo, Japan \\ ${ }^{4}$ Shimabara Observatory, Institute of Seismology and Volcanology, Faculty of Sciences, \\ Kyushu University, Shimabara, Japan \\ ${ }^{5}$ Institute of Seismology and Volcanology, Graduate School of Science, Hokkaido University, Sapporo, Japan \\ ${ }^{6}$ Earthquake Research Institute, The University of Tokyo, Tokyo, Japan \\ ${ }^{7}$ Institute for Research on Earth Evolution, Japan Agency for Marine-Earth Science and Technology, Yokohama, Japan \\ Email: "shigeru@auecc.aichi-edu.ac.jp, miya@sci.kagoshima-u.ac.jp, kanao@nipr.ac.jp, mat@sevo.kyushu-u.ac.jp, \\ takada@sci.hokudai.ac.jp, atsushi@eri.u-tokyo.ac.jp, mikiya@jamstec.go.jp
}

Received December 2, 2013; revised January 1, 2014; accepted February 2, 2014

Copyright (C) 2014 Shigeru Toda et al. This is an open access article distributed under the Creative Commons Attribution License, which permits unrestricted use, distribution, and reproduction in any medium, provided the original work is properly cited. In accordance of the Creative Commons Attribution License all Copyrights (c) 2014 are reserved for SCIRP and the owner of the intellectual property Shigeru Toda et al. All Copyright (C) 2014 are guarded by law and by SCIRP as a guardian.

\begin{abstract}
A gravity survey on Mizuho Plateau was carried out in austral summer of 2001-2002 by the $43^{\text {rd }}$ Japanese Antarctic Research Expedition (JARE-43), as one of the geophysical measurements of deep seismic exploration. Gravity measurements were conducted at 151 points in approximately $1 \mathrm{~km}$ interval along the $150 \mathrm{~km}$ profile. Considering the gravity effect from the ice-sheet, Free-air and Bouguer anomalies were calculated by using precise GPS locations. Furrowed and basin-like negative Free-air anomalies were observed in middle and northern parts of the profile. Bouguer anomalies were calculated by two bedrock elevation data obtained from seismic refraction and radio-echo sounding. High resolution model for bedrock elevation by radio-echo sounding was applied in determining the crustal thickness.
\end{abstract}

\section{KEYWORDS}

\section{Gravity Survey; Mizuho Plateau; Bedrock Elevation; Bouguer Gravity Anomaly; Crustal Thickness}

\section{Introduction}

Several geophysical investigations have been carried out to understand the physical characteristics beneath the continental ice-sheet on Mizuho Plateau, East Antarctica by the Japanese Antarctic Research Expedition (JARE). The Mizuho Plateau is a part of the Paleozoic LützowHolm Complex (LHC), which is known as a region which experienced a metamorphic event in $550 \mathrm{Ma}$ [1]. Before this study, land traverse gravity surveys were conducted for several times on the Plateau since 1961 [2-5]. A significant inclination of the Moho discontinuity from inland plateau area to the coast along the Mizuho-

${ }^{*}$ Corresponding author.
Dome-F routes was analyzed by the previously obtained gravity data [6]. In these previous studies, however, a station spacing of the gravity measurements was about a few $\mathrm{km}$ or larger, therefore it requires that the station interval be more dense to address detail discussion about the fine structure.

A multidisciplinary geosciences project on "Structure and Evolution of the East Antarctic Lithosphere (SEAL)" had been conducted in the Western Enderby LandEastern Dronning Maud Land in a framework of JARE $[7,8]$. As a main component of the SEAL project, the JARE-41 conducted deep seismic exploration (refraction and wide-angle reflection surveys), as well as the gravity measurements with a dense station distribution along the 
Mizuho traverse routes in austral summer of 1999-2000 [9]. A P-wave velocity structure of the ice-sheet and uppermost crustal layer along the profile were obtained by analyzing the recorded travel-time data [10]. [11] obtained gravity anomalies beneath the Mizuho traverse routes. The estimated density model by [11] indicates that the Moho discontinuity slightly declines about $1 \mathrm{~km}$ from coast to inland over $200 \mathrm{~km}$ in length.

In succession to the JARE-41 field operation, a similar seismic exploration was held on the Mizuho Plateau by the JARE-43 in austral summer of 2001-2002. The specifications of the seismic observations and instrumentations were the same as those of JARE-41. The survey profile of JARE-43 intersects the JARE-41 profile at "H176" point and spreads out to NNE-SSW direction with $150 \mathrm{~km}$ in length (Figure 1). More detailed specifications of the JARE-43 seismic surveys are referred to [12]. Dense gravity measurements were also conducted along the JARE-43 profile. In this paper, we represent the details of the gravity measurements, and demonstrate the obtained Free-air and Bouguer gravity anomalies along the survey line by JARE-43.

\section{Gravity Survey}

\subsection{Gravity Measurements}

The land traverse gravity measurements were conducted using a SCINTREX (CG-3M; model: 858011, serial No. 9507278) gravimeter at $1 \mathrm{~km}$ interval along the survey line. The number of measurement points was 151 (Table

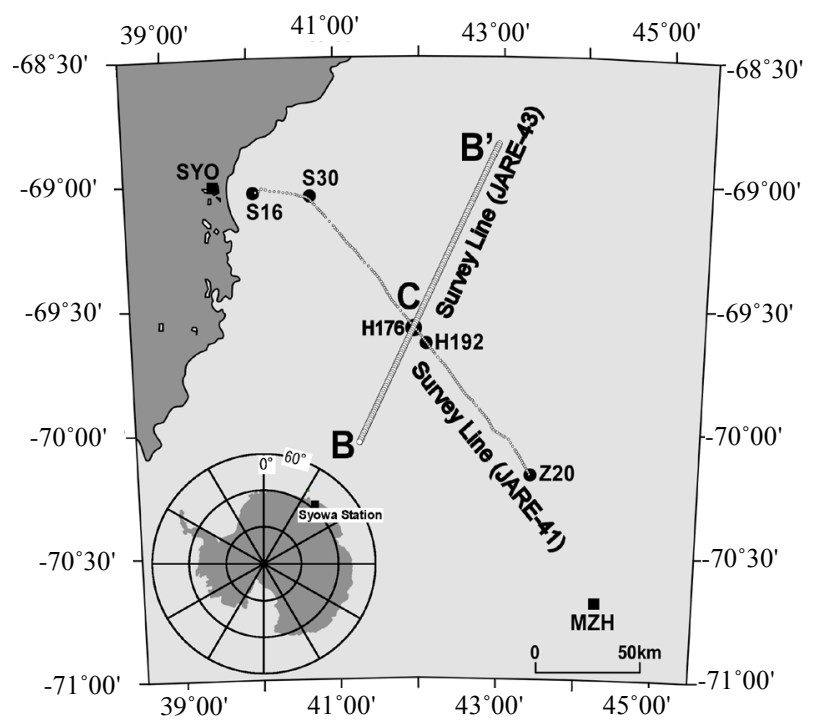

Figure 1. Map showing the JARE-43 gravity survey profile. Small open circles indicate 151 measurement points. Solid line denotes the JARE-41 survey profile. Large solid circles and squares show important route points (S16 and H176) and the Japanese Antarctic Stations (Syowa: SYO; Mizuho: MZH), respectively.
1). Since the survey line was located over $100 \mathrm{~km}$ apart from the gravity reference point at Syowa Station (SYO; $69.0 \mathrm{~S}, 39.6 \mathrm{E}$ ), we set up two temporal gravity reference points at both S16 and H176. These reference points were linked to the absolute gravity point (IAGBN-A) at SYO and gravity values of the points were determined using the absolute gravity value of 982524.327 mgal [13]. In the gravity measurements, we did not adopt the closed loop method which was usually used in conventional gravity surveys. Instead, we carried out the gravity measurements more than twice at several stations including S16 and H176 reference points. If the three successive measurements agreed within a certain allowance revel (10 micro-gal), the final gravity value at the point was determined to be a mean value of these three measurements. The drift rate throughout the total measurement period of 48 days was $0.47 \mathrm{mgal} / \mathrm{day}$ and we found no significant jumps in reading the values. Figure 2 shows the drift rate throughout the measurement period by joining the reading values at S16 and H176.

\subsection{GPS Measurements}

In order to determine precise positions of the gravity points, we used dual-frequency GPS (Ashtech Z type recievers). To determine coordinates of a point, we recorded 20 minutes GPS data for every $5 \mathrm{~s}$ sampling interval. The coordinates of almost the points were precisely calculated on the WGS-84 ellipsoid by rapid static positioning method between the each point and the permanent GPS station at SYO. In contrast, the coordinates of a few numbers of points were calculated by means of "autogipsy" on the basis of the global GPS database (http://gipsy.jpl.nasa.gov). Figure 3 represents the errors in the positioning calculating procedure. Elevation errors in almost all points were achieved less than $0.3 \mathrm{~m}$; which corresponds to the errors in free-air anomalies of about 0.1 mgal.

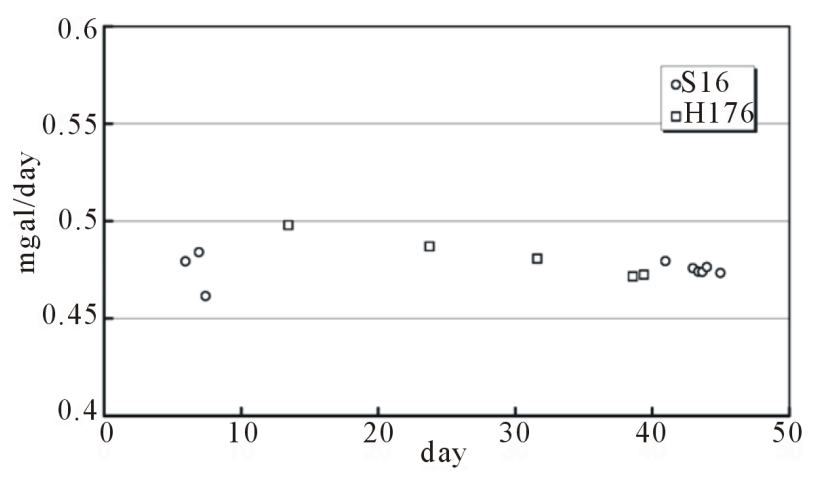

Figure 2. A drift rate throughout the measurement period in 2001-2002 survey. The approximately the same value in $0.47 \mathrm{mgal} /$ day was obtained from both the results at S16 and $\mathrm{H176}$ observation points. 
Table 1. Results of the gravity survey on the Mizuho Plateau in 2001-2002 operation by JARE-43.

\begin{tabular}{|c|c|c|c|c|c|c|c|c|c|c|c|c|c|}
\hline \multirow{2}{*}{ Station } & \multicolumn{3}{|c|}{ Latitude } & \multicolumn{3}{|c|}{ Longitude } & \multirow{2}{*}{$\begin{array}{l}\text { Altitude } \\
\text { (m) }\end{array}$} & \multirow{2}{*}{$\begin{array}{l}\text { Gravity } \\
\text { value } \\
\text { (mgal) }\end{array}$} & \multirow{2}{*}{$\begin{array}{c}\text { Free Air } \\
\text { anomaly } \\
\text { (mgal) }\end{array}$} & \multirow{2}{*}{$\begin{array}{c}\text { Bedrock } \\
\text { elevation } \\
\text { Ice Rader } \\
\text { (m) }\end{array}$} & \multirow{2}{*}{$\begin{array}{l}\text { Bouguer } \\
\text { anomaly } \\
\text { Ice Rader } \\
\text { (mgal) }\end{array}$} & \multirow{2}{*}{$\begin{array}{l}\text { Bedrock } \\
\text { elevation } \\
\text { seismic } \\
\text { (m) }\end{array}$} & \multirow{2}{*}{$\begin{array}{c}\text { Bouguer } \\
\text { anomaly } \\
\text { seismic } \\
\text { (mgal) }\end{array}$} \\
\hline & (deg) & $(\min )$ & (sec) & (deg) & (min) & (sec) & & & & & & & \\
\hline HM001 & 70 & 3 & 51.3 & 41 & 14 & 34.7 & 1481.43 & 982172.66 & 16.9 & & & 0 & -39.0 \\
\hline HM002 & 70 & 3 & 22.5 & 41 & 15 & 17.5 & 1480.92 & 982172.94 & 17.5 & & & 0 & -38.4 \\
\hline HM003 & 70 & 2 & 53.5 & 41 & 16 & 0.5 & 1485.18 & 982171.44 & 17.7 & & & 0 & -38.3 \\
\hline HM004 & 70 & 2 & 24.6 & 41 & 16 & 43.2 & 1488.04 & 982169.22 & 16.9 & & & 0 & -39.3 \\
\hline HM005 & 70 & 1 & 55.8 & 41 & 17 & 26.3 & 1492.05 & 982166.66 & 16.0 & & & -50 & -36.4 \\
\hline HM006 & 70 & 1 & 26.9 & 41 & 18 & 9.1 & 1493.38 & 982164.54 & 14.8 & & & -100 & -33.8 \\
\hline HМ007 & 70 & 0 & 58.1 & 41 & 18 & 51.4 & 1490.85 & 982163.78 & 13.7 & & & -150 & -30.9 \\
\hline HМ008 & 70 & 0 & 29.2 & 41 & 19 & 34.5 & 1490.78 & 982164.38 & 14.8 & & & -150 & -29.9 \\
\hline HМ009 & 70 & 0 & 0.2 & 41 & 20 & 16.9 & 1493.19 & 982165.16 & 16.7 & & & -100 & -31.8 \\
\hline HМ010 & 69 & 59 & 31.4 & 41 & 21 & 0.2 & 1500.75 & 982165.38 & 19.8 & & & -50 & -33.0 \\
\hline HM011 & 69 & 59 & 2.6 & 41 & 21 & 42.6 & 1506.79 & 982166.97 & 23.7 & & & 0 & -33.2 \\
\hline HМ012 & 69 & 58 & 33.8 & 41 & 22 & 24.9 & 1506.15 & 982170.14 & 27.1 & & & 50 & -33.6 \\
\hline HМ013 & 69 & 58 & 5.0 & 41 & 23 & 8.8 & 1505.11 & 982172.22 & 29.3 & & & 100 & -35.2 \\
\hline HМ014 & 69 & 57 & 36.0 & 41 & 23 & 51.1 & 1502.94 & 982173.74 & 30.7 & & & 150 & -37.7 \\
\hline HM015 & 69 & 57 & 7.2 & 41 & 24 & 33.8 & 1497.44 & 982175.06 & 30.8 & & & 150 & -37.4 \\
\hline HМ016 & 69 & 56 & 38.4 & 41 & 25 & 16.0 & 1488.23 & 982177.99 & 31.3 & & & 150 & -36.5 \\
\hline HМ017 & 69 & 56 & 9.5 & 41 & 25 & 58.8 & 1489.43 & 982179.38 & 33.6 & & & 150 & -34.3 \\
\hline HМ018 & 69 & 55 & 40.5 & 41 & 26 & 40.5 & 1490.83 & 982180.59 & 35.7 & & & 150 & -32.2 \\
\hline HМ019 & 69 & 55 & 11.4 & 41 & 27 & 23.1 & 1498.42 & 982177.04 & 34.9 & & & 150 & -33.2 \\
\hline HM020 & 69 & 54 & 42.6 & 41 & 28 & 5.3 & 1512.29 & 982170.20 & 32.8 & & & 150 & -35.9 \\
\hline HМ021 & 69 & 54 & 13.6 & 41 & 28 & 48.1 & 1515.35 & 982165.74 & 29.8 & & & 150 & -39.0 \\
\hline HM022 & 69 & 53 & 44.7 & 41 & 29 & 30.2 & 1516.96 & 982162.41 & 27.4 & & & 100 & -37.6 \\
\hline HМ023 & 69 & 53 & 15.9 & 41 & 30 & 12.7 & 1518.47 & 982158.74 & 24.7 & & & 50 & -36.5 \\
\hline HМ024 & 69 & 52 & 46.9 & 41 & 30 & 55.2 & 1516.81 & 982155.72 & 21.6 & & & 0 & -35.6 \\
\hline HM025 & 69 & 52 & 18.0 & 41 & 31 & 37.3 & 1513.97 & 982154.90 & 20.4 & & & 0 & -36.7 \\
\hline HM026 & 69 & 51 & 48.9 & 41 & 32 & 19.4 & 1511.86 & 982155.02 & 20.4 & & & 0 & -36.7 \\
\hline HM027 & 69 & 51 & 20.1 & 41 & 33 & 1.7 & 1509.17 & 982155.05 & 20.0 & & & 0 & -36.9 \\
\hline HМ028 & 69 & 50 & 51.2 & 41 & 33 & 43.8 & 1509.89 & 982154.11 & 19.8 & & & 0 & -37.2 \\
\hline HM029 & 69 & 50 & 22.3 & 41 & 34 & 26.1 & 1509.54 & 982153.60 & 19.6 & & & 0 & -37.3 \\
\hline HМ030 & 69 & 49 & 53.2 & 41 & 35 & 8.0 & 1511.58 & 982152.65 & 19.8 & & & 0 & -37.2 \\
\hline HM031 & 69 & 49 & 24.3 & 41 & 35 & 50.3 & 1514.72 & 982150.38 & 19.0 & & & 0 & -38.2 \\
\hline HM032 & 69 & 48 & 55.4 & 41 & 36 & 31.6 & 1516.80 & 982148.50 & 18.2 & & & 0 & -39.0 \\
\hline HМ033 & 69 & 48 & 26.5 & 41 & 37 & 14.0 & 1517.97 & 982147.13 & 17.6 & & & 0 & -39.6 \\
\hline HM034 & 69 & 47 & 57.4 & 41 & 37 & 55.8 & 1516.14 & 982146.77 & 17.2 & & & 0 & -40.0 \\
\hline HM035 & 69 & 47 & 28.5 & 41 & 38 & 38.0 & 1514.45 & 982146.87 & 17.3 & & & 0 & -39.9 \\
\hline HМ036 & 69 & 46 & 59.7 & 41 & 39 & 19.5 & 1516.51 & 982146.44 & 17.9 & & & 0 & -39.3 \\
\hline HМ037 & 69 & 46 & 30.5 & 41 & 40 & 1.3 & 1519.57 & 982145.95 & 18.9 & & & 0 & -38.5 \\
\hline HМ038 & 69 & 46 & 1.8 & 41 & 40 & 43.5 & 1517.78 & 982145.67 & 18.5 & & & 0 & -38.8 \\
\hline
\end{tabular}




\section{Continued}

\begin{tabular}{|c|c|c|c|c|c|c|c|c|c|c|c|c|c|}
\hline HM039 & 69 & 45 & 32.7 & 41 & 41 & 24.9 & 1518.99 & 982145.64 & 19.3 & & & 0 & -38.0 \\
\hline HM040 & 69 & 45 & 3.6 & 41 & 42 & 6.9 & 1517.05 & 982146.68 & 20.2 & & & -10 & -36.2 \\
\hline HM041 & 69 & 44 & 34.6 & 41 & 42 & 48.3 & 1516.95 & 982147.48 & 21.5 & & & -20 & -34.4 \\
\hline HM042 & 69 & 44 & 5.6 & 41 & 43 & 30.1 & 1522.00 & 982146.05 & 22.1 & 111 & -43.0 & -30 & -33.3 \\
\hline HM043 & 69 & 43 & 36.6 & 41 & 44 & 11.7 & 1525.17 & 982143.41 & 20.9 & 43 & -39.6 & -40 & -33.9 \\
\hline HM044 & 69 & 43 & 7.7 & 41 & 44 & 53.8 & 1529.98 & 982141.25 & 20.7 & 77 & -42.3 & -50 & -33.6 \\
\hline HM045 & 69 & 42 & 38.6 & 41 & 45 & 35.4 & 1531.50 & 982140.01 & 20.4 & 24 & -39.0 & -60 & -33.2 \\
\hline HM046 & 69 & 42 & 9.8 & 41 & 46 & 17.3 & 1534.12 & 982138.87 & 20.5 & 28 & -39.3 & -70 & -32.5 \\
\hline HM047 & 69 & 41 & 40.7 & 41 & 46 & 58.3 & 1537.46 & 982137.92 & 21.1 & 67 & -41.6 & -80 & -31.4 \\
\hline HM048 & 69 & 41 & 11.6 & 41 & 47 & 40.0 & 1541.28 & 982137.25 & 22.1 & 47 & -39.3 & -90 & -29.8 \\
\hline HM049 & 69 & 40 & 42.7 & 41 & 48 & 21.8 & 1545.37 & 982136.27 & 22.8 & 94 & -41.9 & -100 & -28.5 \\
\hline HM050 & 69 & 40 & 13.6 & 41 & 49 & 2.7 & 1547.32 & 982135.03 & 22.7 & 160 & -46.8 & -100 & -28.8 \\
\hline HM051 & 69 & 39 & 44.6 & 41 & 49 & 44.0 & 1547.96 & 982133.44 & 21.8 & 84 & -42.5 & -90 & -30.4 \\
\hline HM052 & 69 & 39 & 15.6 & 41 & 50 & 25.9 & 1549.11 & 982131.33 & 20.5 & 57 & -41.9 & -80 & -32.4 \\
\hline HM053 & 69 & 38 & 46.5 & 41 & 51 & 7.0 & 1547.01 & 982129.56 & 18.5 & 7 & -40.3 & -70 & -35.0 \\
\hline HM054 & 69 & 38 & 17.6 & 41 & 51 & 48.6 & 1545.26 & 982127.91 & 16.8 & 39 & -44.2 & -60 & -37.3 \\
\hline HM055 & 69 & 37 & 48.6 & 41 & 52 & 29.4 & 1544.69 & 982126.51 & 15.7 & 12 & -43.4 & -50 & -39.1 \\
\hline HM056 & 69 & 37 & 19.5 & 41 & 53 & 10.8 & 1547.78 & 982124.90 & 15.6 & 32 & -45.0 & -50 & -39.4 \\
\hline HM057 & 69 & 36 & 50.4 & 41 & 53 & 52.3 & 1547.49 & 982123.67 & 14.7 & 13 & -44.6 & -50 & -40.2 \\
\hline HM059 & 69 & 36 & 21.4 & 41 & 54 & 33.3 & 1547.06 & 982122.59 & 14.0 & 19 & -45.7 & -50 & -40.9 \\
\hline HM061 & 69 & 35 & 52.4 & 41 & 55 & 14.4 & 1547.99 & 982120.61 & 12.8 & 57 & -49.6 & -50 & -42.2 \\
\hline HM063 & 69 & 35 & 23.5 & 41 & 55 & 55.9 & 1550.24 & 982117.27 & 10.6 & -30 & -45.8 & -50 & -44.4 \\
\hline HM065 & 69 & 34 & 54.3 & 41 & 56 & 37.6 & 1550.96 & 982113.49 & 7.5 & -60 & -46.9 & -150 & -40.6 \\
\hline HM067 & 69 & 34 & 24.9 & 41 & 57 & 16.0 & 1553.44 & 982109.67 & 4.9 & -94 & -47.1 & -250 & -36.4 \\
\hline HM069 & 69 & 33 & 56.5 & 41 & 57 & 59.0 & 1553.80 & 982106.31 & 2.2 & -176 & -44.3 & -350 & -32.2 \\
\hline HM071 & 69 & 33 & 27.5 & 41 & 58 & 40.2 & 1550.59 & 982104.30 & -0.3 & -266 & -40.4 & -450 & -27.7 \\
\hline HM073 & 69 & 32 & 58.5 & 41 & 59 & 21.4 & 1544.32 & 982103.26 & -2.9 & -500 & -26.5 & -550 & -23.1 \\
\hline HM075 & 69 & 32 & 29.4 & 42 & 0 & 2.0 & 1539.90 & 982103.34 & -3.7 & -300 & -41.0 & -550 & -23.7 \\
\hline HM077 & 69 & 32 & 0.4 & 42 & 0 & 43.0 & 1538.63 & 982105.54 & -1.4 & -200 & -45.6 & -450 & -28.3 \\
\hline HM078 & 69 & 31 & 31.3 & 42 & 1 & 24.0 & 1540.19 & 982109.46 & 3.5 & -88 & -48.5 & -350 & -30.4 \\
\hline HM079 & 69 & 31 & 2.2 & 42 & 2 & 4.8 & 1541.09 & 982113.12 & 7.9 & -31 & -48.1 & -250 & -32.9 \\
\hline HM080 & 69 & 30 & 33.2 & 42 & 2 & 45.6 & 1542.40 & 982115.24 & 10.9 & 10 & -47.9 & -150 & -36.9 \\
\hline HM081 & 69 & 30 & 4.1 & 42 & 3 & 26.7 & 1543.00 & 982115.99 & 12.4 & 17 & -47.0 & -100 & -38.9 \\
\hline HM082 & 69 & 29 & 35.0 & 42 & 4 & 7.4 & 1543.18 & 982116.17 & 13.1 & -30 & -43.1 & -100 & -38.2 \\
\hline HM083 & 69 & 29 & 5.9 & 42 & 4 & 48.0 & 1543.04 & 982116.36 & 13.7 & -46 & -41.4 & -100 & -37.6 \\
\hline HM084 & 69 & 28 & 36.9 & 42 & 5 & 28.8 & 1543.01 & 982116.60 & 14.4 & -49 & -40.4 & -100 & -36.9 \\
\hline HM085 & 69 & 28 & 7.9 & 42 & 6 & 9.7 & 1541.04 & 982117.18 & 14.9 & -4 & -43.0 & -100 & -36.4 \\
\hline HM086 & 69 & 27 & 38.7 & 42 & 6 & 50.5 & 1541.52 & 982117.11 & 15.4 & -1 & -42.6 & -100 & -35.8 \\
\hline HM087 & 69 & 27 & 9.6 & 42 & 7 & 30.7 & 1539.34 & 982116.89 & 15.0 & 8 & -43.6 & -100 & -36.1 \\
\hline HM088 & 69 & 26 & 40.7 & 42 & 8 & 11.6 & 1538.05 & 982116.47 & 14.7 & -49 & -40.0 & -50 & -39.9 \\
\hline HM089 & 69 & 26 & 11.5 & 42 & 8 & 52.1 & 1535.83 & 982116.06 & 14.1 & -46 & -40.7 & 0 & -43.9 \\
\hline
\end{tabular}




\section{Continued}

\begin{tabular}{|c|c|c|c|c|c|c|c|c|c|c|c|c|c|}
\hline HM090 & 69 & 25 & 42.5 & 42 & 9 & 32.5 & 1534.79 & 982116.21 & 14.4 & 4 & -43.8 & 10 & -44.2 \\
\hline HM091 & 69 & 25 & 13.3 & 42 & 10 & 12.8 & 1534.00 & 982116.97 & 15.4 & -9 & -41.9 & 20 & -43.9 \\
\hline HM092 & 69 & 24 & 44.4 & 42 & 10 & 53.3 & 1535.50 & 982117.93 & 17.3 & 13 & -41.5 & 30 & -42.7 \\
\hline HM093 & 69 & 24 & 15.3 & 42 & 11 & 33.6 & 1539.70 & 982118.50 & 19.6 & 96 & -45.1 & 40 & -41.2 \\
\hline HM094 & 69 & 23 & 46.2 & 42 & 12 & 14.0 & 1543.21 & 982119.39 & 22.1 & 223 & -51.6 & 50 & -39.6 \\
\hline HM095 & 69 & 23 & 17.0 & 42 & 12 & 54.2 & 1542.25 & 982120.52 & 23.4 & 222 & -50.2 & 40 & -37.5 \\
\hline HM096 & 69 & 22 & 48.0 & 42 & 13 & 34.7 & 1542.55 & 982119.49 & 23.0 & 99 & -42.1 & 30 & -37.3 \\
\hline HM097 & 69 & 22 & 18.9 & 42 & 14 & 14.9 & 1547.09 & 982117.15 & 22.5 & -8 & -35.3 & 20 & -37.3 \\
\hline HM098 & 69 & 21 & 49.8 & 42 & 14 & 55.3 & 1548.96 & 982116.15 & 22.6 & 65 & -40.4 & 10 & -36.6 \\
\hline HM099 & 69 & 21 & 20.8 & 42 & 15 & 35.8 & 1545.24 & 982115.21 & 21.0 & -2 & -37.2 & 0 & -37.3 \\
\hline HM100 & 69 & 20 & 51.7 & 42 & 16 & 15.7 & 1545.05 & 982113.31 & 19.5 & -54 & -35.1 & -10 & -38.1 \\
\hline HM101 & 69 & 20 & 22.2 & 42 & 16 & 56.0 & 1544.12 & 982111.76 & 18.1 & -92 & -33.8 & -20 & -38.7 \\
\hline HM102 & 69 & 19 & 53.1 & 42 & 17 & 35.8 & 1544.66 & 982111.40 & 18.4 & -88 & -33.8 & -30 & -37.8 \\
\hline HM103 & 69 & 19 & 24.1 & 42 & 18 & 15.3 & 1542.02 & 982112.34 & 19.0 & -25 & -37.4 & -40 & -36.4 \\
\hline HM104 & 69 & 18 & 54.8 & 42 & 18 & 56.9 & 1542.57 & 982113.10 & 20.5 & 11 & -38.5 & -50 & -34.3 \\
\hline HM105 & 69 & 18 & 25.8 & 42 & 19 & 36.5 & 1541.85 & 982112.77 & 20.4 & 21 & -39.2 & -60 & -33.6 \\
\hline HM106 & 69 & 17 & 56.7 & 42 & 20 & 16.4 & 1540.93 & 982111.73 & 19.6 & 0 & -38.6 & -70 & -33.7 \\
\hline HM107 & 69 & 17 & 27.5 & 42 & 20 & 56.5 & 1537.29 & 982111.12 & 18.3 & 6 & -40.1 & -80 & -34.2 \\
\hline HM108 & 69 & 16 & 58.4 & 42 & 21 & 36.5 & 1531.40 & 982110.78 & 16.6 & 17 & -42.3 & -90 & -34.9 \\
\hline HM109 & 69 & 16 & 29.3 & 42 & 22 & 16.3 & 1527.85 & 982109.24 & 14.5 & -57 & -39.2 & -90 & -36.9 \\
\hline HM110 & 69 & 16 & 0.1 & 42 & 22 & 56.1 & 1527.84 & 982107.22 & 12.9 & -64 & -40.3 & -80 & -39.2 \\
\hline HM111 & 69 & 15 & 31.0 & 42 & 23 & 35.9 & 1526.91 & 982105.483 & 11.4 & -46 & -43.0 & -70 & -41.4 \\
\hline HM112 & 69 & 15 & 1.9 & 42 & 24 & 16.0 & 1526.72 & 982104.262 & 10.6 & -43 & -44.0 & -60 & -42.8 \\
\hline HM113 & 69 & 14 & 32.8 & 42 & 24 & 55.6 & 1524.70 & 982103.030 & 9.2 & -99 & -41.4 & -50 & -44.8 \\
\hline HM114 & 69 & 14 & 3.8 & 42 & 25 & 35.2 & 1522.35 & 982102.393 & 8.4 & -97 & -42.4 & -40 & -46.3 \\
\hline HM115 & 69 & 13 & 34.6 & 42 & 26 & 15.0 & 1521.31 & 982102.201 & 8.3 & -99 & -42.2 & -30 & -47.0 \\
\hline HM116 & 69 & 13 & 5.5 & 42 & 26 & 54.8 & 1521.06 & 982103.155 & 9.7 & -54 & -43.9 & -20 & -46.3 \\
\hline HM117 & 69 & 12 & 36.1 & 42 & 27 & 34.5 & 1519.11 & 982106.105 & 12.6 & 159 & -55.7 & -10 & -44.1 \\
\hline HM118 & 69 & 12 & 7.0 & 42 & 28 & 14.1 & 1511.40 & 982110.061 & 14.6 & 180 & -54.8 & 0 & -42.4 \\
\hline HM119 & 69 & 11 & 37.9 & 42 & 28 & 54.1 & 1507.74 & 982112.288 & 16.2 & 149 & -51.0 & 10 & -41.4 \\
\hline HM120 & 69 & 11 & 8.6 & 42 & 29 & 33.4 & 1505.56 & 982113.422 & 17.2 & 260 & -57.6 & 20 & -41.0 \\
\hline HM121 & 69 & 10 & 39.5 & 42 & 30 & 13.2 & 1500.14 & 982113.896 & 16.4 & 162 & -51.3 & 30 & -42.2 \\
\hline HM122 & 69 & 10 & 10.5 & 42 & 30 & 52.6 & 1498.78 & 982112.755 & 15.4 & 94 & -47.7 & 40 & -43.9 \\
\hline HM123 & 69 & 9 & 41.4 & 42 & 31 & 32.4 & 1498.80 & 982111.607 & 14.7 & 96 & -48.5 & 50 & -45.3 \\
\hline HM124 & 69 & 9 & 12.2 & 42 & 32 & 12.0 & 1498.56 & 982110.848 & 14.4 & 76 & -47.4 & 60 & -46.3 \\
\hline HM125 & 69 & 8 & 43.1 & 42 & 32 & 51.3 & 1495.38 & 982110.460 & 13.5 & 61 & -47.1 & 70 & -47.8 \\
\hline HM126 & 69 & 8 & 13.8 & 42 & 33 & 30.6 & 1494.97 & 982109.927 & 13.3 & 74 & -48.2 & 80 & -48.6 \\
\hline HM127 & 69 & 7 & 44.6 & 42 & 34 & 10.0 & 1492.44 & 982110.689 & 13.8 & 134 & -51.8 & 90 & -48.7 \\
\hline HM128 & 69 & 7 & 15.4 & 42 & 34 & 49.3 & 1483.77 & 982112.676 & 13.6 & 211 & -57.0 & 100 & -49.3 \\
\hline HM129 & 69 & 6 & 46.3 & 42 & 35 & 28.7 & 1477.42 & 982111.564 & 11.0 & 54 & -48.5 & 0 & -44.7 \\
\hline HM130 & 69 & 6 & 17.2 & 42 & 36 & 8.2 & 1481.02 & 982108.487 & 9.5 & 21 & -47.8 & -100 & -39.4 \\
\hline
\end{tabular}




\begin{tabular}{|c|c|c|c|c|c|c|c|c|c|c|c|c|c|}
\hline \multicolumn{14}{|c|}{ Continued } \\
\hline HM131 & 69 & 5 & 48.0 & 42 & 36 & 47.3 & 1474.92 & 982108.761 & 8.4 & 76 & -52.5 & -150 & -36.8 \\
\hline HM132 & 69 & 5 & 18.9 & 42 & 37 & 26.8 & 1468.02 & 982108.224 & 6.3 & 13 & -50.0 & -200 & -35.3 \\
\hline HM133 & 69 & 4 & 49.6 & 42 & 38 & 6.0 & 1464.64 & 982107.506 & 5.0 & 11 & -51.0 & -200 & -36.4 \\
\hline HM134 & 69 & 4 & 20.3 & 42 & 38 & 45.0 & 1461.16 & 982107.179 & 4.1 & -16 & -49.9 & -200 & -37.2 \\
\hline HM135 & 69 & 3 & 51.0 & 42 & 39 & 24.2 & 1459.68 & 982106.803 & 3.7 & -46 & -48.1 & -200 & -37.5 \\
\hline HM136 & 69 & 3 & 21.7 & 42 & 40 & 3.4 & 1458.36 & 982107.003 & 4.0 & -24 & -49.3 & -200 & -37.2 \\
\hline HM137 & 69 & 2 & 52.7 & 42 & 40 & 42.4 & 1454.28 & 982108.234 & 4.5 & -9 & -49.7 & -200 & -36.5 \\
\hline HM138 & 69 & 2 & 23.5 & 42 & 41 & 21.6 & 1448.60 & 982109.120 & 4.1 & -25 & -48.8 & -200 & -36.7 \\
\hline HM139 & 69 & 1 & 54.2 & 42 & 42 & 0.5 & 1445.78 & 982109.512 & 4.1 & -62 & -46.1 & -250 & -33.1 \\
\hline HM140 & 69 & 1 & 25.1 & 42 & 42 & 39.8 & 1444.90 & 982109.872 & 4.7 & -86 & -43.8 & -300 & -29.0 \\
\hline HM141 & 69 & 0 & 56.0 & 42 & 43 & 18.8 & 1438.80 & 982111.213 & 4.7 & -77 & -44.3 & -300 & -28.9 \\
\hline HM142 & 69 & 0 & 26.6 & 42 & 43 & 57.8 & 1433.41 & 982112.780 & 5.1 & -82 & -43.3 & -300 & -28.3 \\
\hline HM143 & 68 & 59 & 57.4 & 42 & 44 & 36.6 & 1426.48 & 982114.610 & 5.3 & -83 & -42.8 & -300 & -27.8 \\
\hline HM144 & 68 & 59 & 28.3 & 42 & 45 & 15.3 & 1418.87 & 982117.180 & 6.0 & -66 & -43.0 & -300 & -26.8 \\
\hline HM145 & 68 & 58 & 59.1 & 42 & 45 & 54.5 & 1413.31 & 982118.629 & 6.2 & -105 & -39.9 & -300 & -26.4 \\
\hline HM146 & 68 & 58 & 29.8 & 42 & 46 & 33.4 & 1405.54 & 982121.219 & 6.9 & -131 & -37.1 & -300 & -25.4 \\
\hline HM147 & 68 & 58 & 0.6 & 42 & 47 & 12.3 & 1395.89 & 982124.815 & 8.0 & -127 & -35.9 & -300 & -23.9 \\
\hline HM148 & 68 & 57 & 31.4 & 42 & 47 & 51.1 & 1380.02 & 982129.407 & 8.2 & -148 & -33.7 & -300 & -23.1 \\
\hline HM149 & 68 & 57 & 2.2 & 42 & 48 & 30.3 & 1380.86 & 982130.081 & 9.6 & -179 & -30.1 & -300 & -21.7 \\
\hline HM150 & 68 & 56 & 33.0 & 42 & 49 & 8.8 & 1385.28 & 982130.351 & 11.7 & -121 & -32.2 & -300 & -19.8 \\
\hline HM151 & 68 & 56 & 3.7 & 42 & 49 & 47.2 & 1385.62 & 982132.587 & 14.6 & -56 & -33.8 & -300 & -16.9 \\
\hline HM152 & 68 & 55 & 34.5 & 42 & 50 & 26.0 & 1376.05 & 982137.670 & 17.2 & 0 & -34.7 & -300 & -14.0 \\
\hline HM153 & 68 & 55 & 5.3 & 42 & 51 & 4.6 & 1363.90 & 982141.900 & 18.2 & -44 & -30.2 & -250 & -16.0 \\
\hline HM154 & 68 & 54 & 36.0 & 42 & 51 & 43.4 & 1362.05 & 982143.239 & 19.5 & -41 & -29.1 & -200 & -18.1 \\
\hline HM155 & 68 & 54 & 6.7 & 42 & 52 & 21.7 & 1363.06 & 982144.052 & 21.1 & -28 & -28.4 & -150 & -20.0 \\
\hline HM156 & 68 & 53 & 37.4 & 42 & 53 & 0.2 & 1358.70 & 982145.216 & 21.4 & 24 & -31.5 & -100 & -23.0 \\
\hline HM157 & 68 & 53 & 8.3 & 42 & 53 & 38.8 & 1351.77 & 982146.316 & 20.9 & 18 & -31.4 & -50 & -26.7 \\
\hline HM158 & 68 & 52 & 39.1 & 42 & 54 & 17.4 & 1346.07 & 982145.976 & 19.2 & -47 & -28.3 & 0 & -31.5 \\
\hline HM159 & 68 & 52 & 9.7 & 42 & 54 & 56.0 & 1345.29 & 982144.863 & 18.4 & -91 & -26.1 & 50 & -35.8 \\
\hline HM160 & 68 & 51 & 40.6 & 42 & 55 & 34.2 & 1344.27 & 982144.619 & 18.3 & -60 & -28.2 & 100 & -39.3 \\
\hline HM161 & 68 & 51 & 11.3 & 42 & 56 & 13.0 & 1339.19 & 982145.510 & 18.1 & -58 & -28.3 & 100 & -39.3 \\
\hline
\end{tabular}

\section{Gravity Anomaly Calculations}

\subsection{Methods}

The Free-air anomaly $\Delta g$ is calculated by the following formula,

$$
\Delta g=g-\gamma+0.30839 H+0.87-0.0000965 H
$$

where $g$ is the measured gravity value (in mgal), $\gamma$ is the normal gravity defined on the reference ellipsoid 1980 (in mgal), and $H$ is the WGS-84 ellipsoidal height of the gravity point (in meter). Then, the simple Bouguer anomaly $\Delta g^{\prime}$ is calculated by the formula,

$$
\Delta g^{\prime}=\Delta g-z \pi G\left\{\rho_{i}(H-h)+\rho_{c} h\right\},
$$

where $G$ is the Newton's gravitational constant, $\rho_{c}$ is the density of bedrock assumed to be 2.55 or $2.75 \mathrm{~g} / \mathrm{cm}^{3}$ so as to correspond to the final density models; $\rho_{i}$ is the density of the ice assuming as $0.9 \mathrm{~g} / \mathrm{cm}^{3}$, and $h$ is the elevation of the bedrock in meter.

\subsection{Ice-Sheet Surface and Bedrock Elevations}

In order to calculate the simple Bouguer anomaly, it is necessary to remove the effect of the ice-sheet overlaying 


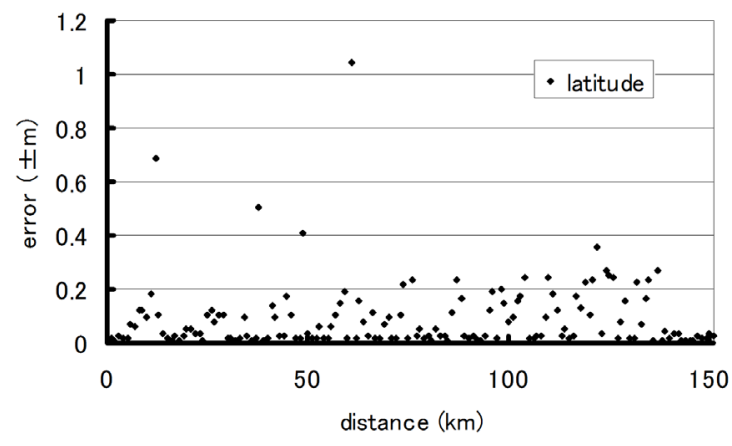

(a)

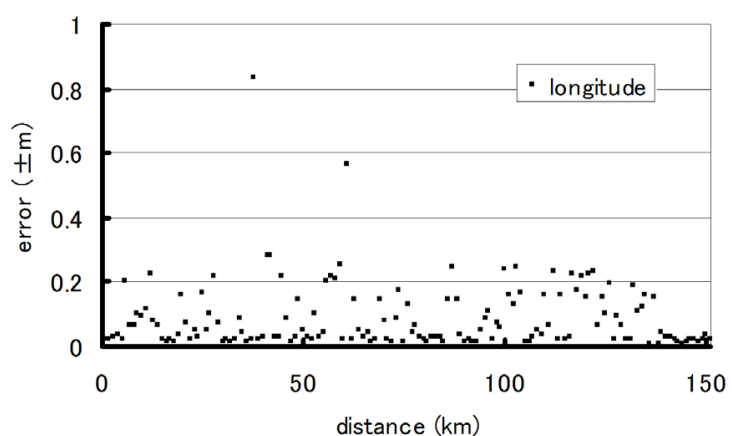

(b)

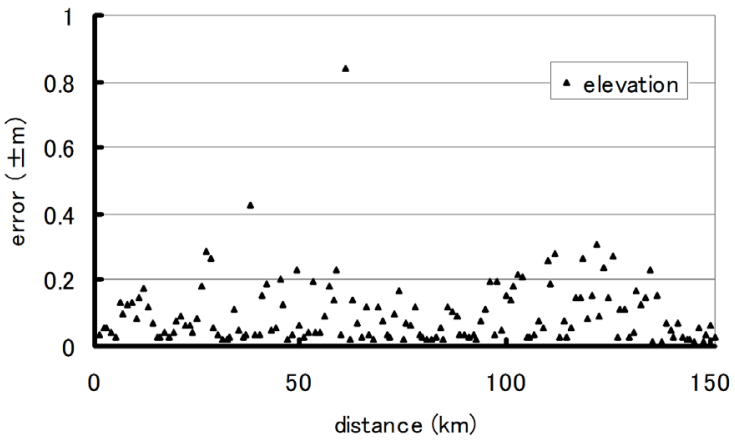

(c)

Figure 3. Calculated GPS positioning errors of all stations. ((a) latitude; (b) longitude; (c) elevation).

the bedrock surface. Two kinds of dataset for the bedrock elevation (that is reduced from ice-sheet surface to the ice-sheet thickness) beneath the survey line are available. The one is a result from the radio-echo sounding simultaneously conducted at the survey ([14], Figure 4), the other is obtained from seismic travel-time analysis [15]. Figure 5 shows the distribution of surface elevation and bedrock elevation of these two data sets. It is clear that the bedrock elevations obtained by seismic travel-time analysis is generally deeper than those obtained by the radio-echo sounding. It is noted that the maximum errors technically anticipated are $100 \mathrm{~m}$ for the radio-echo sounding [16], and $350 \mathrm{~m}$ for the seismic data [10]: These maximum elevation errors could firmly produce the Bouguer anomalies about $7 \mathrm{mgal}$ and $25 \mathrm{mgal}$, respectively.

It is also noted that the bedrock elevations from radioecho sounding were not actually determined at several points, due to a mechanical trouble of the radar instrument. Moreover, there were several places where no reflected signals from the bedrock by the radio-echo sounding, in particular around the points over the furrowed bedrock topography in central part of the profile (point $\mathrm{C}$ in Figure 5, the cross-section between two profiles by JARE-41 and -43). This furrowed bedrock topography was also observed at the Mizuho traverse routes by both the radio-echo sounding and the seismic traveltimes for JARE-41 analyses [10,16]. Unfortunately, it may be difficult to make accurate evaluation for which elevation model could be geophysically and glaciologically more plausible. Therefore, in this paper, we shall calculate the simple Bouguer gravity anomalies by applying for both these two bedrock elevation models.

\subsection{Free-Air and Bouguer Anomalies}

Table 1 shows the coordinates, observed gravity values, obtained Free-air and Bouguer anomalies, together with bedrock elevations at the gravity measured points. Freeair and Bouguer gravity anomalies are also illustrated in Figures 5 and 6, respectively. Figure 5 indicates a fine correlation between the Free-air gravity anomalies and the bedrock topography along the profile. Free-air gravity anomaly varies in +38 and -3 mgal. Furrowed negative free-air anomalies around H176 (point C in Figure 5) implies an existence of a valley structure as seen in the same manner for the bedrock topography. Around this valley, there is no reflected signal recorded from the bedrock by the radio-echo sounding data.

The calculated Bouguer gravity anomalies varies in -10 to -60 mgal (Figure 6). Variation in Bouguer anomalies in adjacent measurement points might be caused chiefly by those of bedrock elevation retrieved from both radio-echo soundings and seismic surveys. Hereafter, therefore, we discuss about the characteristics of Bouger anomalies in long period wave-length over few tens of $\mathrm{km}$ while constructing the crustal density models. The short-period wavelength variations in Bouguer anomalies 


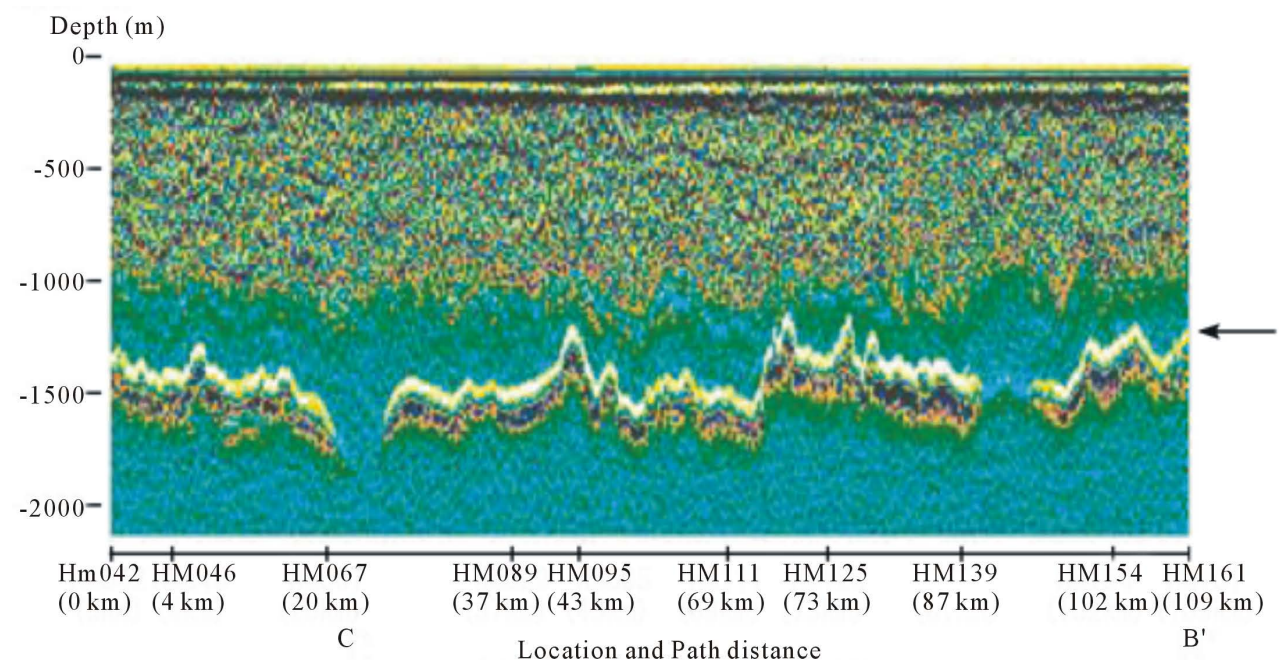

Figure 4. Reflection images of inner structure for the ice-sheet and bedrock topography obtained from radio-echo sounding along the seismic/gravity profile [14]. Black arrow (right hand side in the figure) indicates the reflected echoes from the bedrock surface.

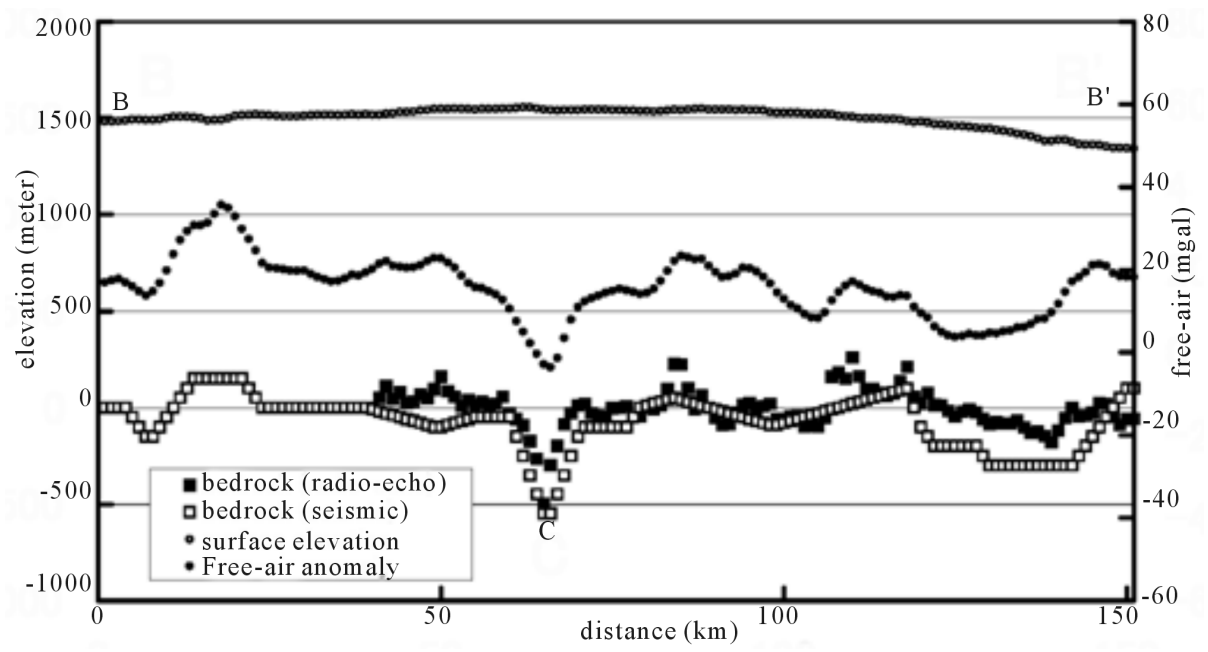

Figure 5. Surface elevation and bedrock topography, together with Free-air gravity anomalies along the survey profile. Small open circles indicate surface elevation determined by GPS measurements. Large solid and open squares indicate the bedrock topography obtained from radio-echo sounding [14] and from the seismic refraction survey [15], respectively. Small solid circles indicate the Free-air gravity anomalies.

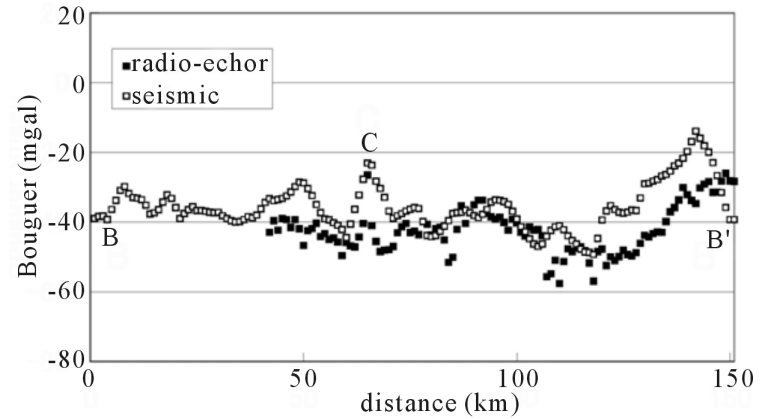

Figure 6. Bouguer gravity anomalies along the survey profile. Solid and open squares indicate the anomalies calculated from the radio-echo sounding [14] and from the seismic refraction survey [15], respectively. supposed to be caused by the small scale heterogeneity in topmost crustal layer including sediments, otherwise a mixture layer composed from ice and moraine rocks spreading over the bedrock beneath continental ice sheet. The negative gravity anomaly suggests the evidence of the thick crust, or the existence of low-density materials are underlying. The Bouguer anomalies in the south and the central parts of the profile have not so much variations and it means that the density discontinuity of the deeper part of the crust could be in less horizontal variation. On the contrary, the low velocity zone in the upper crust at the northern profile ( $\mathrm{B}^{\prime}$ in Figure 6) is implied by the increasing Bouguer anomalies. A "ridge shaped” Bouguer anomalies at H176 (C in Figure 6) is probably 
related to the underlying furrowed bedrock topography.

\section{Discussion}

As a main program of the SEAL deep exploration in austral summer in 2001-2002 by JARE-43, wide-angle reflection and refraction survey was carried out along the seismic profile on the Mizuho Plateau. [15] proposed velocity variations and three prominent seismic velocity boundaries along the profile. The P-wave velocities of the upper crust was identified to have lateral variations of $5.9 \mathrm{~km} / \mathrm{s}$ in the north, $6.0 \mathrm{~km} / \mathrm{s}$ around the point $\mathrm{C}$ (H176), and $6.1-6.2 \mathrm{~km} / \mathrm{s}$ in the center and south of the profile. The first boundary was determined by reflection phases at $19 \mathrm{~km}$ in depth between the upper and the middle crust. The second boundary was recognized by reflected waves from the lower crust at $30 \mathrm{~km}$ in depths. The third boundary was determined by prominent reflection phases from the Moho at $40 \mathrm{~km}$ in depths. However, the inclination of the Moho boundaries are not identified without any significant seismic phase information, because they cannot detect the refracted waves from the Moho (Pn waves) by the experiment caused by the short length less than $200 \mathrm{~km}$ over the whole profile. Shallow part of the crust velocity model by [15] corresponds to the gravity-based model from this study. Lateral variations in P-wave velocities in the upper crust can be explained by assuming the low-density material in the topmost crust. For further study, we might investigate the validity of this boundary's variation by means of a comparison with the other available geophysical data such as geomagnetic anomalies, satellite gravity, together with detail reflection imaging.

\section{Conclusion}

A gravity survey was carried out on the Mizuho Plateau as one of the geophysical measurements by the SEAL exploration in 2001-2002. The gravity measurements were conducted along the seismic profile of $150 \mathrm{~km}$ in length. Free-air and Bouguer anomalies were calculated using the precise locations determined by GPS observations, by considering the effect of thick ice-sheet. The furrowed and basin-like negative Free-air anomalies were obtained in the central and northern parts of the profile. The crustal thickness was demonstrated so as to fit the observed Bouguer anomalies. Our gravimetric results are almost corresponding to the P-wave velocity structure by refraction and wide-angle reflection study [15].

\section{Acknowledgements}

The authors would like to express sincere thanks to the involved members of the JARE-43 seismic survey for their great efforts to carry out the field operations:
Messrs D. Kamiya, T. Nakamura, N. Yoshida, Y. Takahashi, M. Yanagisawa, N. Ishizaki, K. Nakano, T. Yasuhara, S. Iwano and K. Horiguchi. They are also grateful to the expedition members of the JARE-43 (lead by Profs. F. Nishio and the sub leader Prof. K. Kamiyama), members of JARE-42 (the leader Prof. Motoyoshi) and crews of icebreaker "Shirase" (the captain Y. Ishikado). They wish to express their sincere thankfulness to Profs. K. Kaminuma, K. Shibuya, Y. Nogi and other staffs of the National Institute of Polar Research. They would also like to express sincere acknowledgments to Prof. Y. Fukuda of Kyoto University for his fruitful suggestions for analyzing the data.

\section{REFERENCES}

[1] Y. Hiroi, K. Shiraishi and Y. Motoyoshi, "Late Proterozoic Paired Metamorphic Complexes in East Antarctica, with Special Reference to the Tectonic Significance of Ultramafic Rocks,” In: M. R. A. Thomson, et al., Eds., Geological Evolution of Antarctica, Cambridge University Press, Cambridge, 1991, pp. 83-87.

[2] K. Yanai and S. Kaminuma, "Measurement of Gravity along the Traverse Routes Syowa-South Pole,” JARE Sci. Rep., Spec., Vol. 2, 1971, pp. 131-150.

[3] T. Nagao and K. Kaminuma, "Gravity Survey in the Mizuho Plateau,” JARE Data Reports, Vol. 132, 1988, pp. 1-32.

[4] K. Kamiyama, T. Furukawa, H. Maeno, T. Kishi and M. Kanao, "Glaciological Data Collected by the $33^{\text {rd }}$ Japanese Antarctic Research Expedition in 1992,” JARE Data Reports, Vol. 194, 1994, pp. 1-67.

[5] T. Higashi, M. Kanao, H. Motoyama and T. Yamanouchi, "Gravity Observations along the Traverse Routes from Syowa Station to Dome Fuji Station, East Antarctica," Polar Geoscience, Vol. 14, 2001, pp. 226-234.

[6] M. Kanao, K. Kamiyama and K. Ito, "Crustal Density Structure of the Mizuho Plateau, East Antarctica from Gravity Survey in 1992,” Polar Geoscience, Vol. 7, 1994, pp. 23-36.

[7] M. Kanao, M. Ishikawa, M. Yamashita, K. Kaminuma and L. D. Brown, "Structure and Evolution of the East Antarctic Lithosphere: Tectonic Implications for the Development and Dispersal of Gondwana,” Gondwana Research, Vol. 7, No. 1, 2004, pp. 31-41. http://dx.doi.org/10.1016/S1342-937X(05)70304-X

[8] M. Kanao, A. Fujiwara, H. Miyamachi, S. Toda, M. Tomura, K. Ito and T. Ikawa, "Reflection Imaging of the Crust and the Lithospheric Mantle in the Lützow-Holm Complex, Eastern Dronning Maud Land, Antarctica, Derived from the SEAL Transects," Tectonophysics, Vol. 508, No. 1-4, 2011, pp. 73-84. http://dx.doi.org/10.1016/j.tecto.2010.08.005

[9] H. Miyamachi, H. Murakami, T. Tsutsui, S. Toda, T. Minta and M. Yanagisawa, "A Seismic Refraction Experiment in 2000 on the Mizuho Plateau, East Antarctica (JARE-41)," Antarctic Report, Vol. 45, 2001, pp. 101147. 
[10] T. Tsutsui, H. Murakami, H. Miyamachi, S. Toda and M. Kanao, "P-Wave Velocity Structure of the Ice Sheet and the Shallow Crust Beneath the Mizuho Traverse Routes, East Antarctica, from Seismic Refraction Analysis," Polar Geoscience, Vol. 14, 2001, pp. 195-211.

[11] S. Toda, H. Miyamachi, H. Murakami, T. Tsutsui and M. Kanao, "Gravity Survey along the Mizuho Traverse Routes, East Antarctica: SEAL Seismic Exploration in 1999-2000," International Journal of Geosciences, Vol. 4, No. 10, 2013, pp. 1392-1400. http://dx.doi.org/10.4236/ijg.2013.410136

[12] H. Miyamachi, S. Toda, T. Matsushima, M. Takada, Y. Takahashi, D. Kamiya, A. Watanabe, M. Yamashita and M. Yanagisawa, "A Seismic Refraction and Wide-Angle Reflection Exploration in 2002 on the Mizuho Plateau, East Antarctica,” Antarctic Report, Vol. 47, 2003, pp. 3271.

[13] K. Kaminuma, K. Tsukahara and S. Takemoto, “Absolute
Gravity Value Measured at Syowa Station, Antarctica," Bulletin of the D'Information BGI, Vol. 80, 1997, pp. 2629.

[14] M. Takada, S. Toda, D. Kamiya and H. Miyamachi, “The Ice Radar Survey along the JARE-43 Seismic Exploration Profile in the Mizuho Plateau, East Antarctica," Antarctic Report, Vol. 47, 2003, pp. 380-394.

[15] H. Miyamachi, S. Toda,' T. Matsushima, M. Takada, A. Watanabe, M. Yamashita and M. Kanao, "Seismic Refraction and Wide-Angle Reflection Exploration by JARE43 on Mizuho Plateau, East Antarctica," Polar Geoscience, Vol. 16, 2003, pp. 1-21.

[16] H. Maeno, K. Kamiyama, T. Furukawa, O. Watanabe, R. Naruse, K. Okamoto, T. Suitz and S. Uratsuka, "Using a Mobile Radio Echo Sounder to Measure Bedrock Topography in East Queen Maud Land, Antarctica,” Polar Meteorology and Glaciology, Vol. 8, 1994, pp. 149-160. 